\title{
Complex permittivity and conductivity of poly(p-phenylenediazo- methine) and its blends at microwave frequencies
}

\author{
K G PRINCY*, RANI JOSEPH ${ }^{\dagger}$, HONEY JOHN ${ }^{\dagger}$ and K T MATHEW ${ }^{\dagger \dagger}$ \\ Department of Chemistry, Carmel College, Mala 680 732, India \\ ${ }^{\dagger}$ Department of Polymer Science and Rubber Technology, ${ }^{\dagger \dagger}$ Department of Electronics, \\ Cochin University of Science and Technology, Cochin 682 022, India
}

MS received 17 December 2008; revised 18 February 2009

\begin{abstract}
Poly( $p$-phenylenediazomethine) was synthesized by the condensation reaction between glyoxal and $p$-phenylene diamine in different solvents like methanol, toluene, $m$-cresol and $N, N$-dimethylformamide. The dielectric properties and microwave conductivity of the pelletized samples were measured using cavity perturbation technique. The measurements were done at $2.17 \mathrm{GHz}$ at room temperature $\left(25^{\circ} \mathrm{C}\right)$. The effect of dopants on the dielectric properties and conductivity was studied using $\mathrm{HCl}$ and $\mathrm{HClO}_{4}$. Dielectric properties like dielectric constant, dielectric loss factor and microwave conductivity increased on doping with $\mathrm{HCl}$ and $\mathrm{HClO}_{4}$. Conducting polymer composites were prepared by in situ polymerization of glyoxal and p-phenylenediamine in different solvents containing different amounts of PVC, and silica. The microwave conductivity and complex permittivity of each sample was measured. The effect of dopants like $\mathrm{HClO}_{4}$ and HCl on these dielectric properties was also studied. The d.c. conductivity of the pressed samples measured by the two-probe method was also studied.
\end{abstract}

Keywords. Poly(p-phenylenediazomethine); d.c. conductivity; microwave conductivity; complex permittivity; doping; composites.

\section{Introduction}

Microwave properties of conducting polymers is crucial because of their wide areas of applications such as coating in reflector antennas, coating in electronic equipments, frequency selective surfaces, EMI materials, satellite communication links, microchip antennas, and medical applications (Lakshmi et al 2008). Conducting polymers are excellent microwave absorbers and they show technological advantage when compared with inorganic electromagnetic absorbing materials, being light weight, easily processable, and the ability of changing the electromagnetic properties with nature and amount of dopants, synthesis conditions, etc and can be used for making microwave absorbers in space applications (Honey John et al 2007a). Conducting polymers have emerged as a very important class of materials because of their unique electrical, optical and chemical properties leading to the wide range of technological applications. This class of materials provide tremendous scope for tuning of their electrical conductivity from semiconducting to metallic regime by way of doping (MacDiarmid and Epstein 1994; Wessling 1999). They have widespread applications in rechargeable batteries, electrochromic

*Author for correspondence (princykgjohn@gmail.com) devices, gas separation membranes, and above all as a strong EMI shielding materials (Diaz et al 1979, 1981; Peres and Depaoli 1992; Bhat and Yasmin 1995; Pellegrino et al 1996; Kizilyar et al 1999). All dielectric materials are characterized by their dielectric parameters such as dielectric constant, conductivity and dielectric loss factor (Honey John et al 2007b). These parameters differ with frequency, temperature, pressure, etc.

Conducting polymer composites have drawn considerable interest in recent years because of their numerous applications in a variety of areas of electrical and electronic industry (Kanazawa et al 1979, 1980; Biju Kumar et al 2001). A number of groups have reported studies on the electrical conductivity and dielectric properties of composites of a variety of conducting polymers (Yoon et al 1995; Yang et al 1996; Gangopadhyay et al 2001; Murugesan and Subramanian 2003; Tanwar et al 2006). Preparation of blends, composites and interpenetrating network has been widely used as an approach to combine electrical conductivity with desirable mechanical strength of polymers (Honey John et al 2002; Pant et al 2006; Samui et al 2006).

Poly(azomethine)s, sometimes called poly(schiff bases), are a group of polymers which are catching more attention due to the following reasons. Aromatic poly(azomethine)s are highly thermo-stable in analogy to aromatic polyethers. Furthermore, poly(azomethine)s may possess a 
conjugated main chain and, after suitable doping, may show an attractive level of electric conductivity (Kricheldorf and Schwarz 1992).

In the present study, a new conducting polymer based on glyoxal and $p$-phenylene diamine was synthesized. The synthesis of $\operatorname{poly}(p$-phenylenediazomethine) was carried out in different solvents, like, methanol, toluene, $m$-cresol and DMF. First the reaction was carried out in methanol. Since the conductivity of the product formed was very low, the polymerization was repeated in toluene solvent. In this medium also, the conductivity of the product formed was very low and the reaction was repeated in $m$-cresol. Even though the conductivity of the product was higher than other samples already prepared, yield of the product was very low. So the polymerization was carried out in DMF as the solvent.

The dielectric properties and conductivity of the pelletized samples were measured using cavity perturbation technique (Honey John et al 2002). The measurements were done at $2 \cdot 17 \mathrm{GHz}$ at room temperature $\left(25^{\circ} \mathrm{C}\right)$. The effect of dopants on the dielectric properties and conductivity was studied using $\mathrm{HCl}$ and $\mathrm{HClO}_{4}$. Dielectric properties like dielectric constant, dielectric loss factor and microwave conductivity increased on doping with $\mathrm{HCl}$ and $\mathrm{HClO}_{4}$.

Conducting polymer composites with PVC and silica was prepared by in situ polymerization of glyoxal and $p$-phenylenediamine in different solvents containing different amounts of PVC and silica. The microwave conductivity and complex permittivity of each sample was measured. The effect of dopants like $\mathrm{HClO}_{4}$ and $\mathrm{HCl}$ on these dielectric properties was also studied.

\section{Experimental}

\subsection{Materials}

$P$-phenylene diamine, glyoxal $40 \%$ solution, glyoxal hydrate (trimer), $N, N$-dimethyl formamide (DMF), $m$ cresol, toluene, methanol, polyvinyl chloride, ultrafine silica, tetrahydrofuran, acetone, hydrochloric acid $(\mathrm{HCl})$, and perchloric acid $\left(\mathrm{HClO}_{4}\right)$ were used.

\subsection{Synthesis of poly(p-phenylenediazomethine) in methanol}

$0 \cdot 1 \mathrm{~mol}$ of $p$-phenylene diamine was dissolved in methanol $(300 \mathrm{ml})$ and $0.1 \mathrm{~mol}$ glyoxal (40\% solution) was added drop wise with continuous stirring for $2 \mathrm{~h}$. By that time, precipitation of the product took place. The product was washed with water and then with methanol and dried well at room temperature under vacuum.

\subsection{Synthesis of poly(p-phenylenediazomethine) in toluene}

$0.1 \mathrm{~mol}$ of $p$-phenylene diamine was added to $400 \mathrm{ml}$ of toluene and was dissolved by stirring. Then $0 \cdot 1 \mathrm{~mol}$ of glyoxal was added to it and stirred well. The mixture was slowly heated to boiling, with continuous stirring, and was refluxed at boiling temperature for $5 \mathrm{~h}$. By that time, the precipitation of the product took place. It was then allowed to cool to room temperature and then poured into excess of ice-cold water. It was allowed to settle, filtered, washed with plenty of water until the filtrate was colourless. Then it was washed with methanol, and allowed to dry at room temperature in air for one day. Then the final drying of the sample was done at $70^{\circ} \mathrm{C}$ under vacuum.

\subsection{Synthesis of poly(p-phenylene diazomethine) in m-cresol}

$0.1 \mathrm{~mol}$ of $p$-phenylene diamine was added to $100 \mathrm{ml}$ of $m$-cresol and was dissolved by stirring. Then $0.1 \mathrm{~mol}$ of glyoxal was added to it and stirred well. The mixture was slowly heated to boiling, with continuous stirring, and was refluxed at a boiling temperature for $4 \mathrm{~h}$. It was then allowed to cool to room temperature and then poured into excess of ice-cold water. The precipitate was allowed to settle, filtered, washed with plenty of water until the filtrate was colourless. Then it was washed with methanol, and allowed to dry at room temperature in air for one day. Then the final drying of the sample was done at $70^{\circ} \mathrm{C}$ under vacuum.

\subsection{Synthesis of poly(p-phenylenediazomethine) in $N, N$-dimethyl-formamide}

$0.1 \mathrm{~mol}$ of $p$-phenylene diamine was added to $300 \mathrm{ml}$ of DMF and was dissolved by stirring. Then $0.1 \mathrm{~mol}$ of glyoxal was added to it and stirred well. The mixture was slowly heated to boiling, with continuous stirring, and was refluxed at a boiling temperature for $4 \mathrm{~h}$. By that time, the precipitation of the product takes place. It was then allowed to cool to room temperature and then poured into excess of ice-cold water. It was allowed to settle, filtered, washed with plenty of water until the filtrate was colourless. Then it was washed with methanol, and allowed to dry at room temperature in air for one day. Then the final drying of the sample was done at $70^{\circ} \mathrm{C}$ under vacuum.

\subsection{Synthesis of poly(p-phenylenediazomethine)- polyvinylchloride conducting polymer blends}

PVC (5 g) was dissolved in $50 \mathrm{ml}$ of tetrahydrofuran. $0.1 \mathrm{~mol}$ of $p$-phenylene diamine was added to $300 \mathrm{ml}$ of $N, N$-dimethylformamide and was dissolved in it by stirring. Then $0 \cdot 1 \mathrm{~mol}$ of glyoxal was added to it and stirred well. The solution of PVC in THF was added to the above reaction mixture and stirred well. Then the temperature of the reaction medium was increased slowly to 
boiling, with continuous stirring, and was refluxed at a boiling temperature for $4 \mathrm{~h}$. By that time, the precipitation of the product takes place. It was then allowed to cool to room temperature and then poured into excess of ice-cold water with stirring. It was allowed to settle, filtered, washed with plenty of water until the filtrate was colourless. Then it was washed with methanol, and allowed to dry at room temperature in air for one day. Then the final drying of the sample was done at $70^{\circ} \mathrm{C}$ in vacuum. The same procedure was repeated by varying the amount of PVC.

\subsection{Synthesis of poly(p-phenylenediazomethine)- silica conducting polymer composites}

$0.1 \mathrm{~mol}$ of $p$-phenylene diamine was added to $300 \mathrm{ml}$ of DMF and was dissolved in it by stirring. $5 \mathrm{~g}$ of ultrafine silica was added to it and stirred well. Then $0.1 \mathrm{~mol}$ of glyoxal was added to it and stirred well. Then the temperature of the reaction medium was increased slowly to boiling, with continuous stirring, and was refluxed at a boiling temperature for $4 \mathrm{~h}$. By that time, the precipitation of the product takes place. It was then allowed to cool to room temperature and then poured into excess of ice-cold water with stirring. It was allowed to settle, filtered, washed with plenty of water until the filtrate was colourless. Then it was washed with methanol, and allowed to dry at room temperature in air for one day. Then the final drying of the sample was done at $70^{\circ} \mathrm{C}$ in vacuum. The same procedure was repeated by varying the amount of silica to the reaction mixture containing glyoxal and $p$-phenylenediamine in DMF.

\subsection{Doping}

$1 \mathrm{~g}$ of each sample was added in $50 \mathrm{ml}$ each of $1 \mathrm{M} \mathrm{HCl}$ solution and $1 \mathrm{M} \mathrm{HClO}_{4}$ solution and kept for $24 \mathrm{~h}$. Then it was filtered, washed with a little amount of acetone and dried at $70^{\circ} \mathrm{C}$ under dynamic vacuum for $1 \mathrm{~h}$.

\section{Measurement}

\subsection{Microwave conductivity and complex permittivity}

The dielectric properties and conductivity of the samples were measured using cavity perturbation technique (Honey John et al 2002). The experimental set up consists of a HP8510 vector network analyzer; sweep oscillator, S-parameter test set and rectangular cavity resonator. The measurements were done at $2.17 \mathrm{GHz}$ at room temperature $\left(25^{\circ} \mathrm{C}\right)$.

In microwave studies the conductivity can be calculated using the equation

Conductivity, $\sigma=2 \pi f_{\mathrm{s}} \varepsilon_{0} \varepsilon_{\mathrm{r}}^{\prime \prime}$, where $f_{\mathrm{s}}$ is the resonant frequency, $\varepsilon_{0}$ the complex permittivity of free space and $\varepsilon_{\mathrm{r}}^{\prime \prime}$ the imaginary part of the complex permittivity, which is given by the equation,

$$
\varepsilon_{\mathrm{r}}^{\prime \prime}=\left(V_{\mathrm{c}} / 4 V_{\mathrm{s}}\right)\left(Q_{\mathrm{t}}-Q_{\mathrm{s}} / Q_{\mathrm{t}} Q_{\mathrm{s}}\right),
$$

where, $V_{\mathrm{c}}$ is the volume of cavity, $V_{\mathrm{s}}$ the volume of sample, $Q_{\text {s }}$ the quality factor of the cavity loaded with the sample and $Q_{\mathrm{t}}$ the quality factor of the cavity with empty sample holder.

The real part of the complex permittivity, $\varepsilon_{\mathrm{r}}^{\prime}$, is usually known as dielectric constant of the material. It can be calculated from the equation

$$
\varepsilon_{\mathrm{T}}^{\prime}=1+\left(f_{\mathrm{t}}-f_{\mathrm{s}}\right) / 2 f_{\mathrm{s}}\left(V_{\mathrm{c}} / V_{\mathrm{s}}\right),
$$

where $f_{\mathrm{t}}=$ resonant frequency of the unloaded cavity and $f_{\mathrm{s}}$ the resonant frequency of the cavity loaded with the sample.

The pelletized samples were inserted into the cavity and the perturbation produced at a particular microwave frequency was noted. The resonant frequency and quality factor were recorded and dielectric constant and microwave conductivity were calculated using the above equations.

\subsection{D.C. conductivity}

D.C. conductivity of the pressed pellets was determined by the two-probe technique. The samples were sandwiched between two copper electrodes and a constant voltage $(6 \mathrm{~V})$ was applied to the sample. The current flowing through the sample was measured using a digital multimeter (APLAB model 1087). The conductivity of the sample was calculated using the equation

$$
\sigma=t / R A,
$$

where $t$ is the thickness of the pellet, $R$ the resistance of the sample $(R=E / I$, where $E$ is the applied voltage and $I$ the resulting current through the sample), and $A$ the area of cross-section of the pellet $\left(A=\pi r^{2}\right.$, where $r$ is the diameter of the pellet).

\section{Results and discussion}

\subsection{Poly(p-phenylenediazomethine)}

Figures 1-3 show the variation in the dielectric constant, dielectric loss factor, and microwave conductivity of poly ( $p$-phenylenediazomethine) prepared in different solvents at a microwave frequency of $2.17 \mathrm{GHz}$ at $25^{\circ} \mathrm{C}$. The microwave conductivity was less than that of a conductor, but greater than that of an insulator at microwave frequencies. Because of this property, it can be used to construct microwave components like filters.

The real part of the complex permittivity, i.e. dielectric constant of the material, was increased when they were 
doped with $\mathrm{HCl}$ and $\mathrm{HClO}_{4}$. On doping, due to the presence of additional charge carriers, and restriction of their movement in the polymer chain, local accumulation of charge will induce its image charge on an electrode and give rise to interfacial polarization. So dielectric constant was increased on doping, as shown in figure 1. Dielectric constant of the material doped with $\mathrm{HClO}_{4}$ was more than that of undoped and $\mathrm{HCl}$ doped samples. This may be due to the effect of introducing more charge carriers, because, $\mathrm{HClO}_{4}$ is a strong oxidizing agent than $\mathrm{HCl}$.

Figure 2 shows the variation in the imaginary part of the complex permittivity, i.e. dielectric loss factor of different doped and undoped samples. Imaginary part of the complex permittivity or the dielectric loss factor, $\varepsilon_{\mathrm{r}}^{\prime \prime}$ is a direct function of relaxation process and the origin of this relaxation is due to the local motion of polar groups. Dielectric loss factor of the material was increased on doping except for the polymer prepared in methanol. The increase in dielectric loss factor may be due to the increase in the polarity of the molecule on doping. For the large molecule, the rotary motion of the molecules was not sufficiently rapid for the attainment of equilibrium with the field. So more time was required for the dipole to reorient, and hence dielectric loss factor was increased. For the polymer prepared in methanol, the molecular weight of the polymer was very low due to the precipitation of the product, and the reaction was carried out at room temperature. So the time for the molecule to reorient with the field was low and hence on doping, the dielectric loss factor was decreased. Microwave conductivity is a direct function of dielectric loss factor. Hence, the increase in conductivity on doping was due to the increase in dielectric loss factor of the material as shown in figure 3.

The d.c. conductivities of the prepared samples are given table 1. Conductivity of the undoped samples was changed with the solvent in which the reaction was carried out. The variation in conductivity may be due to the difference in the degree of polymerization in different solvents. The conductivity of the doped samples was

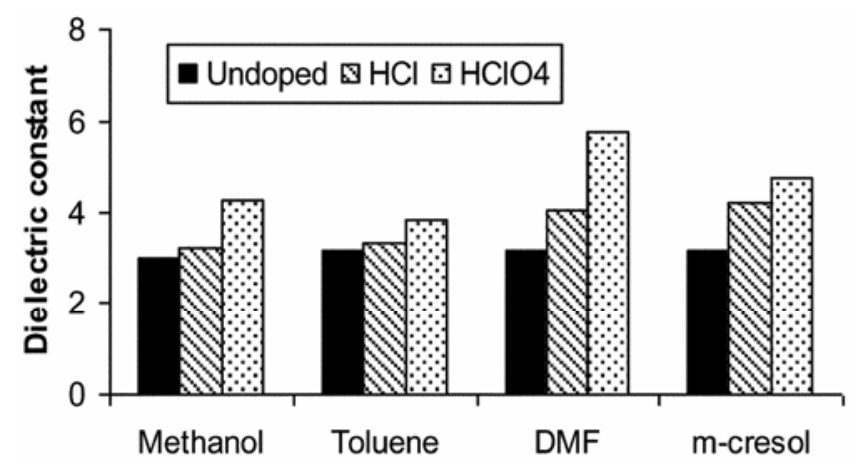

Figure 1. Dielectric constant of $\operatorname{poly}(p$-phenylenediazomethine) prepared in different solvents. more than that of the undoped samples. This was because, after doping, more charge carriers were introduced into the polymer chain, which helped to increase the conductivity. This may be due to the attachment of $\mathrm{H}^{+}$ions to the $\mathrm{N}$ atom of the polymer using the lone pair of electrons, which results in the oxidation of the polymer chain. Charge delocalization on the polymer chain helped to increase the conductivity.

\subsection{Poly(p-phenylenediazomethine)-polyvinylchloride blends}

Dielectric properties of conducting polymer/PVC blends are given in figures $4-6$ at room temperature in the

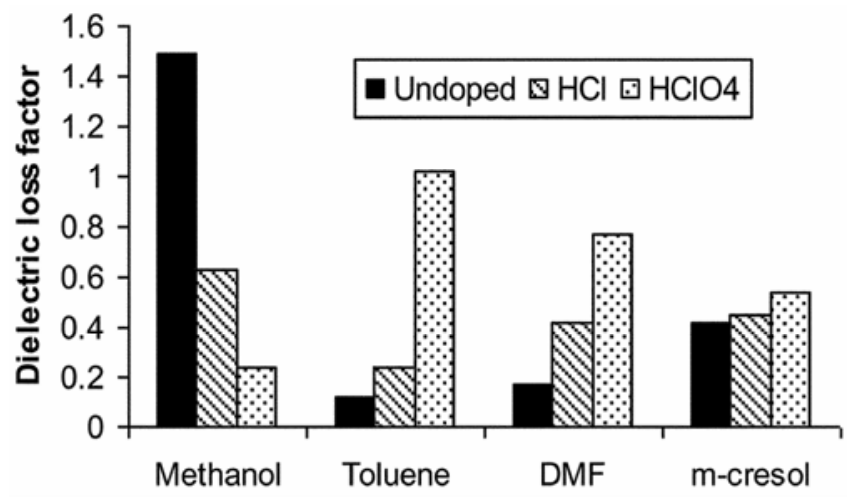

Figure 2. Dielectric loss factor of poly( $p$-phenylenediazomethine) prepared in different solvents.

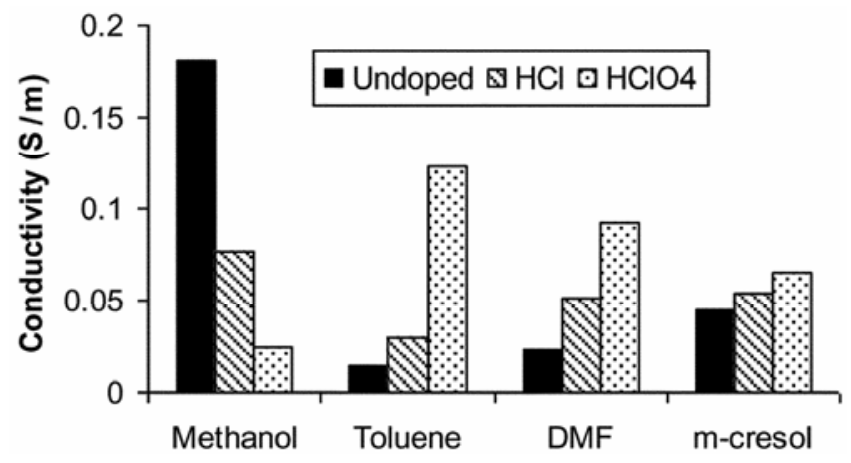

Figure 3. Microwave conductivity of poly( $p$-phenylenediazomethine) prepared in different solvents.

Table 1. Conductivity of $\operatorname{poly}(p$-phenylenediazomethine) prepared in different solvents.

\begin{tabular}{llcc}
\hline & \multicolumn{3}{c}{ Conductivity (S/m) } \\
\cline { 2 - 4 } Solvent & Undoped & \multicolumn{2}{c}{ Dopants } \\
\cline { 2 - 4 } & & $\mathrm{HCl}$ & $\mathrm{HClO}_{4}$ \\
\hline DMF & $1 \cdot 164 \times 10^{-4}$ & $8.604 \times 10^{-2}$ & $2 \cdot 801 \times 10^{-3}$ \\
$m$-Cresol & $2.415 \times 10^{-5}$ & $3.663 \times 10^{-2}$ & $6 \cdot 061 \times 10^{-3}$ \\
Toluene & $6.25 \times 10^{-6}$ & $2.463 \times 10^{-4}$ & $9.709 \times 10^{-3}$ \\
Methanol & $3.597 \times 10^{-8}$ & $2.198 \times 10^{-5}$ & $8.422 \times 10^{-5}$ \\
\hline
\end{tabular}


microwave frequency of $2 \cdot 17 \mathrm{GHz}$. Figure 4 shows the variation in dielectric constant of acid doped and undoped PVC blends. The dielectric constant of the $\mathrm{HCl}$ doped blends were more than that of undoped samples. On doping, due to the presence of additional charge carriers, and their movements which were restricted in the polymer chain, local accumulation of charge will induce its image charge on an electrode and give rise to interfacial polarization. Dielectric constant of $\mathrm{HClO}_{4}$ doped samples was less than that of undoped samples. This may be due to orientation polarization of the dipoles caused by the alternating accumulation of charges at the interfaces between different phases of materials, i.e. between PVC and $\operatorname{poly}(p$-phenylenediazomethine), even though more charge carriers were introduced. As the amount of PVC was increased, dielectric constant was increased first and up to about $10 \mathrm{~g}$ of PVC was added in the blend. This was due to the presence of additional charge carriers, i.e. $\mathrm{HCl}$ eliminated from $\mathrm{PVC}$, which act as dopant. As the amount of PVC was increased more, dielectric constant was decreased due to the insulating effect of PVC.

Figure 5 shows the variation in the imaginary part of the complex permittivity, i.e. dielectric loss factor of the

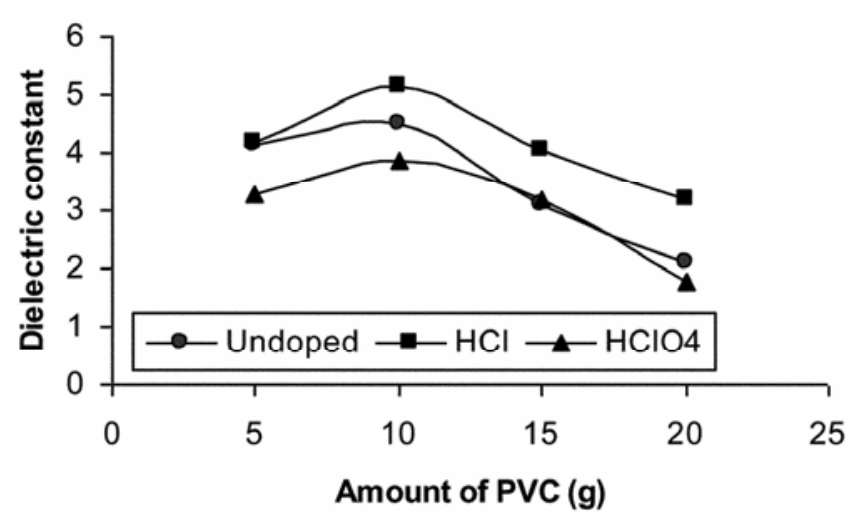

Figure 4. Dielectric constant of conducting polymer/PVC blends.

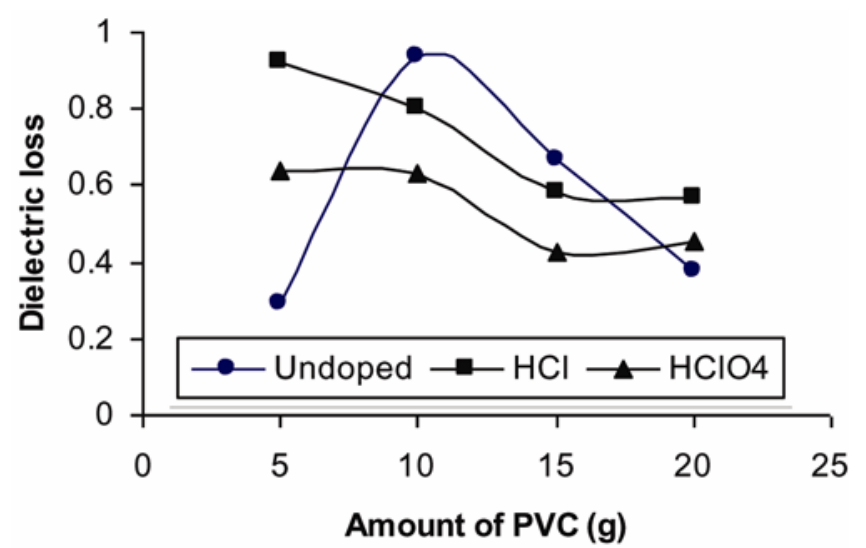

Figure 5. Dielectric loss factor of conducting polymer/PVC blends. conducting polymer/PVC blends. As the amount of PVC was increased, dielectric loss factor of undoped material was increased first, and then decreased. The initial increase in loss may be due to the presence of polar group in PVC in which some of the $\mathrm{HCl}$ eliminated during the polymerization may be acting as a dopant. As the amount of PVC was increased, the insulating effect of PVC predominates, and dielectric loss factor was decreased with increase in the amount of PVC. For doped samples, the dielectric loss factor of the blends was decreased with increase in the amount of PVC. On doping, more charge carriers were introduced, and so the dielectric loss factor of the doped samples were higher than undoped sample containing lower amount of PVC. But as the amount of PVC was increased more, the insulating effect of PVC predominates, and hence dielectric loss factor was decreased.

Figure 6 shows the variation in the microwave conductivity of doped and undoped samples of conducting polymer/PVC blends with the amount of PVC. As the amount of PVC was increased, the microwave conductivity of undoped samples was increased first and then

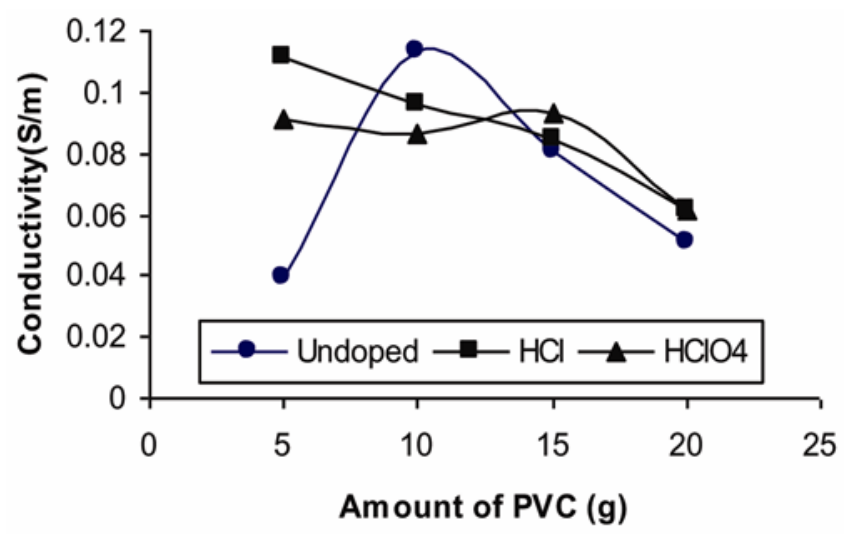

Figure 6. Microwave conductivity of conducting polymer/ PVC blends.

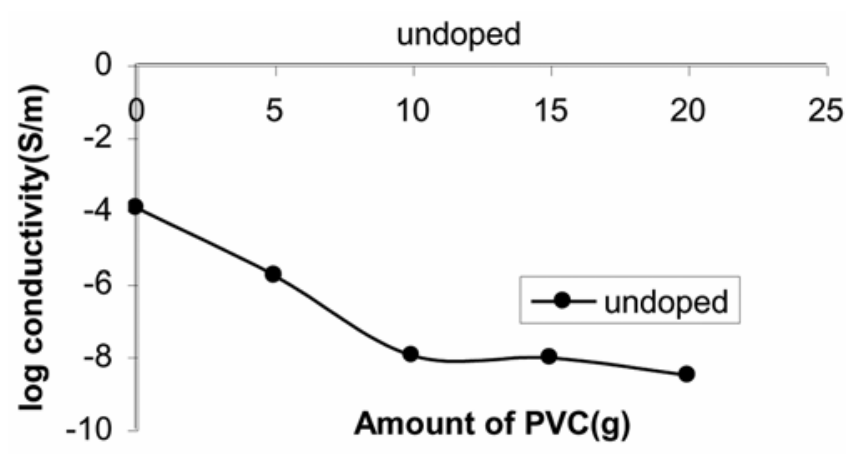

Figure 7. D.C. conductivity of the conducting polymer/PVC blends. 
decreased. This was due to the initial increase and then decrease in the dielectric loss factor of the blends with the amount of PVC. Microwave conductivity is a direct function of dielectric loss. So the change in the microwave conductivity was the same as that of dielectric loss factor. For acid doped samples, the conductivity was decreased as the amount of PVC increased due to the increase in the insulating effect of PVC.

D.C. conductivity of the blends of the conducting polymer based on glyoxal, $p$-phenylene diamine and varying amounts of polyvinyl chloride is shown in figure 7. The d.c. conductivity of the undoped blends decreased with increase in the amount of PVC. It was because, PVC is an insulator and the presence of nonconducting PVC in the composite prevented the free movement of electrons through the conducting polymer chain. Here, the conducting region was separated by a nonconducting region, so that the jumping of electrons from one conducting region to other became difficult.

The variation in d.c. conductivity of the doped samples with the amount of PVC is given in figure 8 . The d.c. conductivity of the doped samples was more than that of the undoped samples. This was because, after doping, more charge carriers were introduced into the polymer chain, which helped to increase the conductivity. Conductivity of perchloric acid doped samples containing higher amount of PVC were more than that of $\mathrm{HCl}$ doped samples. $\mathrm{HClO}_{4}$ was found to be the better doping agent because of the large size of $\mathrm{ClO}_{4}^{-}$counter ion, which helped to tightly attach the dopant to the polymer chain. This may be due to the attachment of $\mathrm{H}^{+}$ions to the $\mathrm{N}$ atom of the polymer using the lone pair of electrons to increase the charge delocalization.

The d.c. conductivity of perchloric acid doped blends were more than that of the pure conducting polymer doped with perchloric acid. As the amount of PVC was increased, d.c. conductivity also increased and remained almost constant at higher amount of PVC. This was because, PVC present in the blend is polar in nature, and

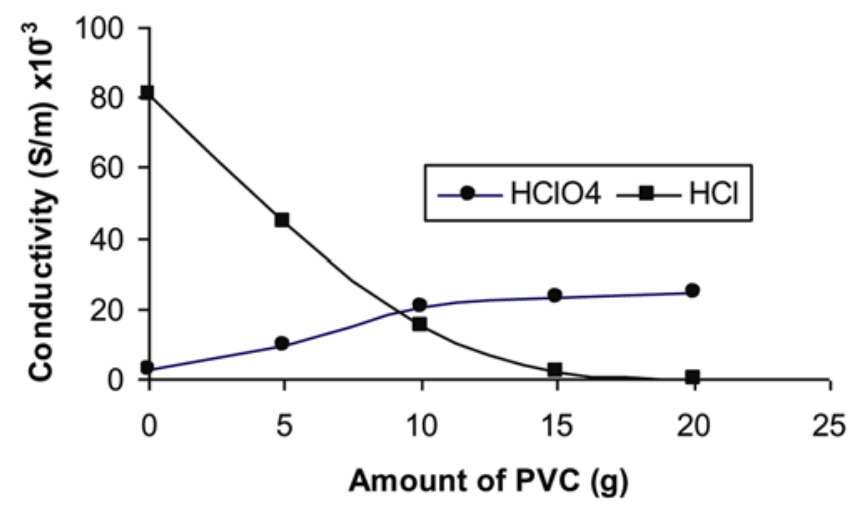

Figure 8. D.C. conductivity of $\mathrm{HCl}$ and $\mathrm{HClO}_{4}$ doped conducting polymer/PVC blends the charge delocalization and oxidation takes place with the conducting polymer as well as with PVC molecules. After a saturation point was reached, the increase in conductivity was negligible because, the amount of insulating region of PVC was increased in the blend.

\subsection{Poly(p-phenylenediazomethine)-silica composites}

The changes in the dielectric parameters of silica composites at $2.17 \mathrm{GHz}$ at room temperature are given in figures 9-11. The variation in dielectric constant for the doped and undoped samples is shown in figure 9. Dielectric constant of $\mathrm{HClO}_{4}$ doped samples was more than that of undoped and $\mathrm{HCl}$ doped samples. It was due to the presence of moisture in the samples due to the hygroscopic nature of silica and $\mathrm{HClO}_{4}$. Hence as the amount of silica was increased, dielectric constant was increased for $\mathrm{HClO}_{4}$ doped samples. For undoped and $\mathrm{HCl}$ doped samples, dielectric constant was decreased with increase in the amount of silica due to the polarization caused by the alternating accumulation of charges at the interfaces between different phases of materials due to the orientation polarization of the dipoles. At higher amount of

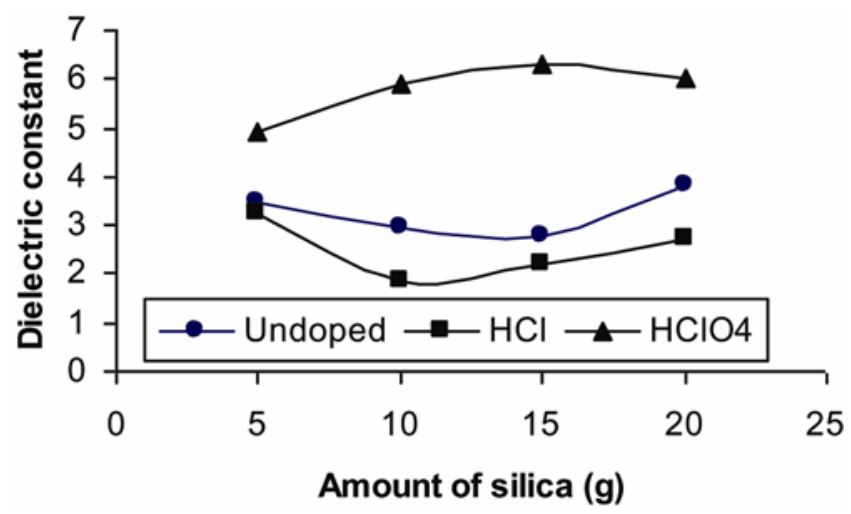

Figure 9. Dielectric constant of conducting polymer/silica composites.

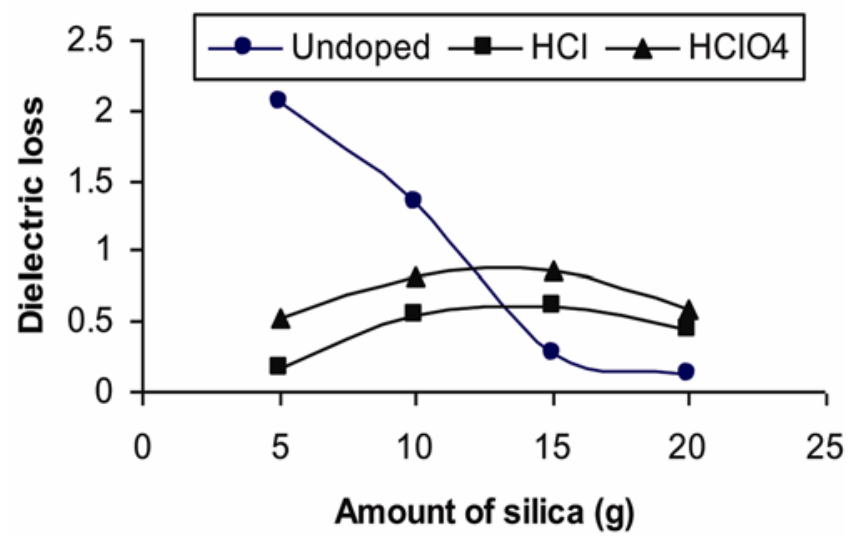

Figure 10. Dielectric loss factor of conducting polymer/silica composites. 
silica, the slight increase in dielectric constant may be due to the moisture present in the sample.

Figures 10 and 11 show the variation in dielectric loss factor and microwave conductivity of the silica composites. For undoped samples, dielectric loss and conductivity decreased as the amount of silica was increased. This was due to the insulating effect of silica. For doped samples, dielectric loss factor and hence the microwave conductivity increased at first, with increase in the amount of silica, reached a maximum value, and then decreased. The increase in the values was due to the presence of moisture in the samples. At higher amount of silica, the insulating effect was predominated and hence microwave conductivity and dielectric loss factor decreased.

The d.c. conductivity of the undoped composites was decreased with increase in the amount of silica similar to the observation for the composites with PVC and is shown in figure 12. It was because silica, which is an insulator, prevents the free movement of electrons through the conducting polymer chain. Here also, a nonconducting region separates the conducting region, so that the jumping of electrons from one conducting region to other becomes difficult. For $\mathrm{HCl}$ doped samples also, conductivity decreased with the amount of silica in the composite as shown in figure 13.

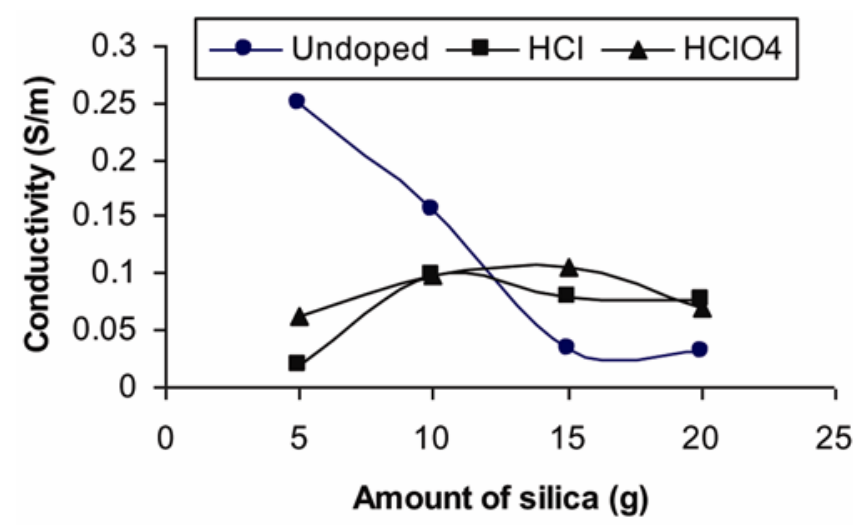

Figure 11. Microwave conductivity of conducting polymer/ silica composites.

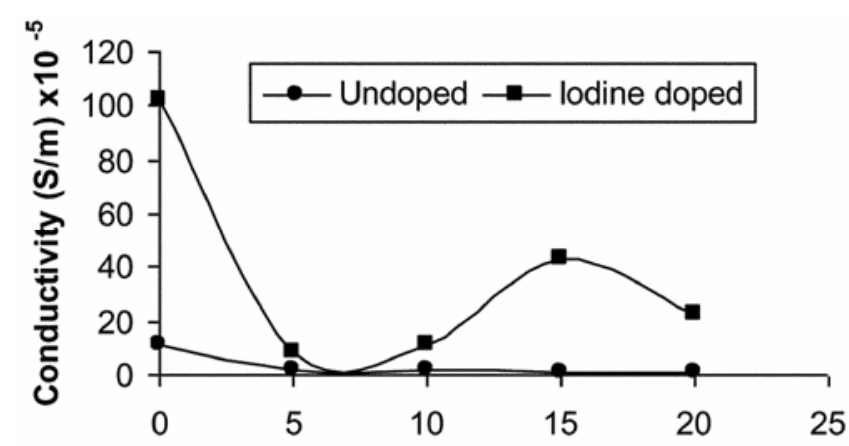

Figure 12. D.C. conductivity of undoped conducting polymer/ silica composites.
The d.c. conductivity of perchloric acid doped composites were more than that of the pure conducting poymer doped with perchloric acid and as the amount of silica was increased, the conductivity increased first up to about $15 \mathrm{~g}$ of silica present in the composite. As the amount of silica was increased further, conductivity decreased due to insulating nature of silica. The conduction process can be accounted for in terms of electrons hopping between localized states under the assistance of proton transfer, for which the presence of water plays an essential role. Silica as well as perchloric acid can adsorb water molecules and the presence of moisture increased the d.c. conductivity.

\section{Conclusions}

(I) Condensation reaction took place between glyoxal and $p$-phenylene diamine to form the conducting polymer, $\operatorname{poly}(p$-phenylenediazomethine).

(II) Dielectric properties like dielectric constant, dielectric loss and microwave conductivity increased on doping with $\mathrm{HCl}$ and $\mathrm{HClO}_{4}$.

(III) Dielectric constant of the material doped with $\mathrm{HClO}_{4}$ was more than that of undoped and $\mathrm{HCl}$ doped samples.

(IV) On doping with $\mathrm{HCl}$, the dielectric constant of the conducting polymer blend with PVC was increased. Dielectric constant was decreased when the samples were doped with $\mathrm{HClO}_{4}$.

(V) As the amount of PVC was increased, the dielectric constant, dielectric loss factor and microwave conductivity of undoped conducting polymer blend with PVC was increased first and then decreased and their values decreased for doped samples.

(VI) For silica composites, dielectric constant of $\mathrm{HClO}_{4}$ doped samples was more than undoped and $\mathrm{HCl}$ doped samples.

(VII) For undoped silica composites, dielectric loss and conductivity decreased as the amount of silica was increased. For doped samples, dielectric loss factor and

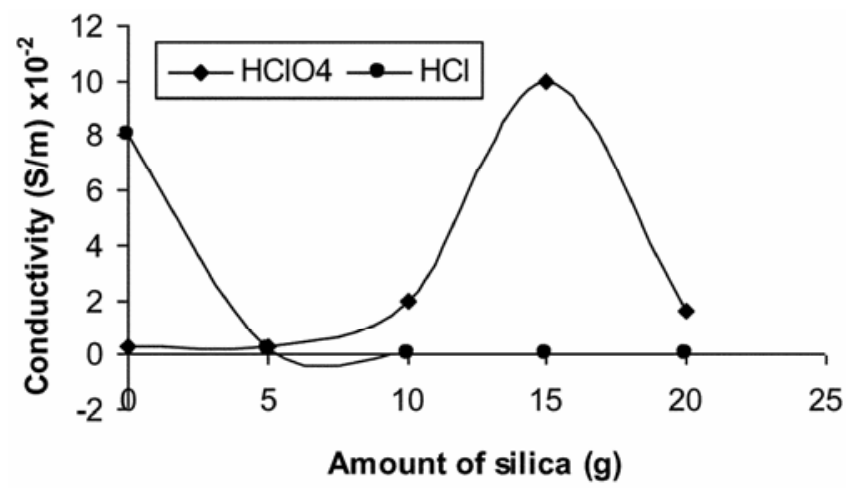

Figure 13. D.C. conductivity of $\mathrm{HCl}$ and $\mathrm{HClO}_{4}$ doped conducting polymer/silica composites. 
hence the microwave conductivity increased at first, with increase in the amount of silica, reached a maximum value, and then decreased.

(VIII) As the amount of silica was increased, dielectric constant was increased for $\mathrm{HClO}_{4}$ doped samples. For undoped and $\mathrm{HCl}$ doped samples, dielectric constant was decreased with increase in the amount of silica.

(IX) For undoped samples, dielectric loss and conductivity decreased as the amount of silica was increased.

(X) For doped samples, dielectric loss factor and hence the microwave conductivity increased at first, with increase in the amount of silica, reached a maximum value, and then decreased.

(XI) D.C. conductivity of the polymer prepared in DMF was more than that of other samples prepared in $m$-cresol, methanol and toluene.

(XII) Doping increased the d.c. conductivity, $\mathrm{HClO}_{4}$ was a better doping agent for poly( $p$-phenylenediazomethine) compared to $\mathrm{HCl}$.

(XIII) The d.c. conductivity of the undoped conducting polymer blends was lower than the d.c. conductivity of the conducting polymer and was decreased with increase in the amount of PVC, and silica.

(XIV) The d.c. conductivity of the conducting polymer blends was increased on doping with $\mathrm{HCl}, \mathrm{HClO}_{4}$.

\section{References}

Bhat N V and Yasmin S 1995 J. Appl. Polym. Sci. 551827

Biju Kumar S, Honey John, Rani Joseph, Hajian M, Ligthart L P and Mathew K T 2001 J. Eur. Ceram. Soc. 212677

Diaz A F, Kanazawa K K and Gardini G P 1979 J. Chem. Soc. Chem. Commun. 635
Diaz A F, Castillo J I, Logan T A and Lee W J 1981 Electroanal. Chem. 129115

Gangopadhyay R, De A and Ghosh G 2001 Synth. Met. 123529

Honey John, Biju Kumar S, Mathew K T and Rani Joseph 2002 J. Appl. Polym. Sci. $\mathbf{8 3} 2008$

Honey John, Rani Joseph and Mathew K T 2007a J. Appl. Polym. Sci. 1032682

Honey John, Rinku M Thomas, Joe Jacob, Mathew K T and Rani Joseph 2007b Polym. Compos. 28588

Kanazawa K K, Diaz A F, Gill W D, Grant P M, Street G B, Gardini G P and Kwak J F 1979/1980 Synth. Met. 329

Kizilyar N, Ozden N Y, Toppare L and Yagci Y 1999 Synth. Met. 10445

Kricheldorf H R and Schwarz G 1992 Handbook of polymer synthesis (New York: Marcel Dekker) Part B, Ch. 27, p. 1673

Lakshmi K, Honey John, Rani Joseph, George K E and Mathew K T 2008 Microwave Opt. Technol. Lett. 50504

MacDiarmid A G and Epstein A J 1994 Frontiers of polymers and advanced materials (New York: Plenum Press) p. 251

Murugesan R and Subramanian E 2003 Bull. Mater. Sci. 26529

Pant H C, Patra M K, Negi S C, Bhatia A, Vadera S R and Kumar N 2006 Bull. Mater. Sci. 29379

Pellegrino J, Radebaugh R and Mattes B R 1996 Macromolecules 294985

Peres R C D and DePaoli M A 1992 J. Power Sources 40229

Samui A B, Manoj N R, Raut R and Patankar A S 2006 J. Appl. Polym. Sci. 1011217

Tanwar A, Gupta K K, Singh P J and Vijay Y K 2006 Bull. Mater. Sci. 29181

Wessling B 1999 Synth. Met. 1021396

Yang J, Hou J, Zhu W, Xu M and Wan M 1996 Synth. Met. 80 283

Yoon C O, Reghu M, Moses D, Cao Y and Heeger A J 1995 Synth. Met. 26255 